\title{
ALMA reveals a large structured disk and nested rotating outflows in DG Tauri B
}

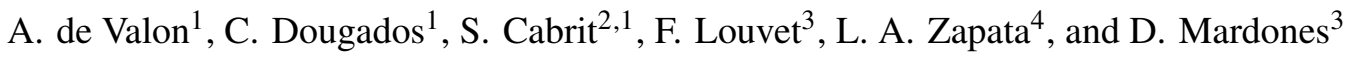 \\ 1 Univ. Grenoble Alpes, CNRS, IPAG, 38000 Grenoble, France \\ e-mail: alois.de-valon@univ-grenoble-alpes.fr \\ 2 PSL University, Sorbonne Université, Observatoire de Paris, LERMA, CNRS UMR 8112, 75014 Paris, France \\ 3 Departamento de Astronomía de Chile, Universidad de Chile, Santiago, Chile \\ ${ }^{4}$ Instituto de Radioastronomía y Astrofíica, Universidad Nacional Autónoma de México, PO Box 3-72, 58090 Morelia, \\ Michoacán, Mexico
}

Received 18 October 2019 / Accepted 15 January 2020

\section{ABSTRACT}

\begin{abstract}
We present Atacama Large Millimeter Array (ALMA) Band 6 observations at 14-20 au spatial resolution of the disk and CO(2-1) outflow around the Class I protostar DG Tau B in Taurus. The disk is very large, both in dust continuum $\left(R_{\mathrm{eff}, 95 \%}=174 \mathrm{au}\right)$ and CO $\left(R_{\mathrm{CO}}=700 \mathrm{au}\right)$. It shows Keplerian rotation around a $1.1 \pm 0.2 M_{\odot}$ central star and two dust emission bumps at $r=62$ and 135 au. These results confirm that large structured disks can form at an early stage where residual infall is still ongoing. The redshifted CO outflow at high velocity shows a striking hollow cone morphology out to 3000 au with a shear-like velocity structure within the cone walls. These walls coincide with the scattered light cavity, and they appear to be rooted within $<60$ au in the disk. We confirm their global average rotation in the same sense as the disk, with a specific angular momentum $\simeq 65$ au km s${ }^{-1}$. The mass-flux rate of $1.7-2.9 \times 10^{-7} M_{\odot} \mathrm{yr}^{-1}$ is $35 \pm 10$ times that in the atomic jet. We also detect a wider and slower outflow component surrounding this inner conical flow, which also rotates in the same direction as the disk. Our ALMA observations therefore demonstrate that the inner cone walls, and the associated scattered light cavity, do not trace the interface with infalling material, which is shown to be confined to much wider angles $\left(>70^{\circ}\right)$. The properties of the conical walls are suggestive of the interaction between an episodic inner jet or wind with an outer disk wind, or of a massive disk wind originating from 2 to $5 \mathrm{au}$. However, further modeling is required to establish their origin. In either case, such massive outflow may significantly affect the disk structure and evolution.
\end{abstract}

Key words. stars: formation - ISM: jets and outflows - protoplanetary disks - stars: individual: DG Tau B

\section{Introduction}

Jets and outflows are ubiquitous at all stages of star formation. However, their exact origin and impact on mass and angular momentum extraction are still fundamental open questions (Frank et al. 2014). Slow molecular outflows are traditionally interpreted as swept-up material, tracing the interaction of the jet or a wide-angle wind with the infalling envelope and parent core (e.g., in Zhang et al. 2016). Recent interferometric observations with the Atacama Large Millimeter Array (ALMA) have revealed, in a few cases, rotation levels suggesting that material directly ejected from the disk, through photo-evaporation or magneto-centrifugal processes, might also perhaps contribute to the formation of low velocity molecular cavities on small scales. These rotating cavities have been observed at all stages from Class 0 (e.g., HH212, IRAS 4C in Tabone et al. 2018; Zhang et al. 2018), Class I (e.g., TMCIA in Bjerkeli et al. 2016), and Class II stars (HH30 in Louvet et al. 2018). If present, such disk winds could play a crucial role in the transport of angular momentum through the disk and the final dispersion of gas. Studies at high-angular and spectral resolution of objects with weak or no infalling envelopes with ALMA are crucial to help discriminate between an envelope and a disk origin for the rotating outflow material.

DG Tau B is a highly reddened Class I/II protostar located in the dark globule B217 in the Taurus complex (Schmalzl et al. 2010). Gaia DR2 parallaxes of optically visible young stars in the same globule ${ }^{1}$ suggest a distance $\simeq 140 \mathrm{pc}$, which we adopt thereafter. This object drives an asymmetric bipolar jet at $\mathrm{PA}=122^{\circ}$ (Mundt et al. 1991), which was first discovered by Mundt \& Fried (1983) and studied in detail by Podio et al. (2011). Asymetric conical scattering nebulae have also been observed at optical and near-infrared wavelengths (Padgett et al. 1999) with half opening angles of respectively $42^{\circ}$ (blue lobe) and $22^{\circ}$ (red lobe). A slow asymmetrical molecular outflow was first mapped in $\mathrm{CO}$ by Mitchell et al. (1997), which was mainly associated to the receding atomic jet. Mottram et al. (2017) show that both CO lobes extend up to at least $60^{\prime \prime}(8400 \mathrm{au})$ with opening angles similar to the infrared scattering nebulae. They also show the absence of $\mathrm{HCO}+$ emission on source, which is typical for young stars with weak envelopes (van Kempen et al. 2009). Zapata et al. (2015) studied DG Tau B with the Submillimeter Array (SMA) at 2" angular resolution and they report velocity asymmetries across the receding conical $\mathrm{CO}$ outflow interpreted as rotation signatures. They propose that the massive redshifted conical outflow traces entrained material from the rotating parent core.

In this Letter we present continuum, ${ }^{12} \mathrm{CO}(2-1),{ }^{13} \mathrm{CO}(2-1)$, and $\mathrm{C}^{18} \mathrm{O}(2-1)$ observations acquired with ALMA of the

1 Excluding DG Tau A where bright nebulosity introduces an unusually high measurement error for its $G$ magnitude (Brown et al. 2018). 

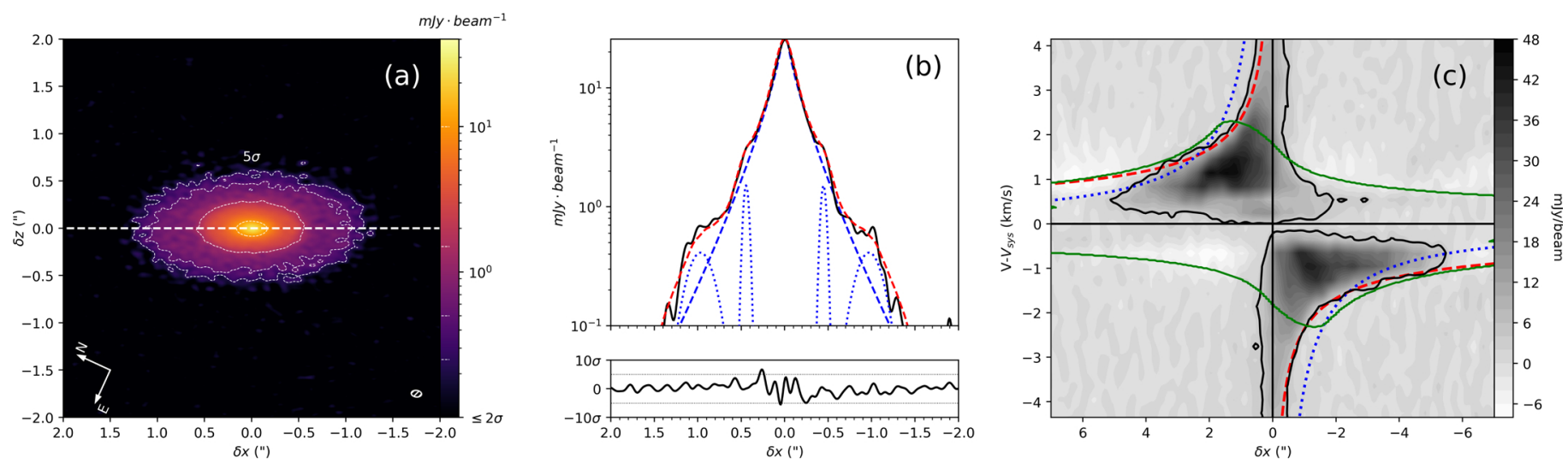

Fig. 1. (a) Continuum map at $232 \mathrm{GHz}$ in logarithmic scale. White dotted contours show the $5,10,30$, and $200 \sigma$ levels $\left(\sigma=50 \mu \mathrm{Jy}\right.$ beam $\left.{ }^{-1}\right)$. The white dashed line shows the disk major axis at $\mathrm{PA}=25.7^{\circ}$. (b) Continuum intensity profiles: cut along $\mathrm{PA}=25.7^{\circ}$ (in black) and deprojected and azimuthally averaged (in red). The tapered power law central component and the two Gaussian components, derived from the fitting and before beam convolution, are shown in blue dashed and dotted lines, respectively. Bottom panel: fit residuals and $\pm 5 \sigma$ limit (dotted lines). (c) Positionvelocity diagram in ${ }^{13} \mathrm{CO}$ along $\mathrm{PA}=25.7^{\circ}$ in a pseudo-slit of $4^{\prime \prime}$ width. The $3 \sigma$ contour is shown in black. The curves show maximum expected line-of-sight velocities $V_{\max }$ for three different models: a thin disk in Keplerian rotation around $M_{\star}=1.1 M_{\odot}$ at an inclination of $i=63^{\circ}$ (red dashed line), pure rotation with constant angular momentum $V_{\max } \propto 1 / r$ (blue dotted line), and a rotating and infalling bipolar shell (see Sect. 3.2.2) with $\mathrm{Rd}=300 \mathrm{au}, \theta_{0}=70^{\circ}$ at an inclination of $i=63^{\circ}$ (green solid line).

DG tau B disk and outflow, which increase the angular resolution by more than a factor of 10 with respect to the previous study of Zapata et al. (2015). In Sect. 2 we describe the observations and data reduction. In Sect. 3 we present new results revealing a large and structured disk as well as a rotating outer wide angle component, surrounding the inner conical flow. In Sect. 4 we discuss new implications for the origin of disk structure and small-scale conical CO outflows.

\section{Observations and data reduction}

DG Tau B was observed in band 6 during ALMA Cycles 3 and 5 with three $12 \mathrm{~m}$ array configurations covering a baseline range of $11-6500 k \lambda(15-8500 \mathrm{~m})$. The details of the observations are presented in Table A.1. The spectral set-up was similar for each observation and included three spectral windows centered on the ${ }^{12} \mathrm{CO}(2-1),{ }^{13} \mathrm{CO}(2-1)$, and $\mathrm{C}^{18} \mathrm{O}(2-1)$ emission lines, each with a native spectral resolution of $122 \mathrm{kHz}$, which we rebinned to $244 \mathrm{kHz}\left(\approx 0.3 \mathrm{~km} \mathrm{~s}^{-1}\right)$ to improve sensitivity. An additional spectral window with $2 \mathrm{GHz}$ bandwidth centered at $\simeq 232 \mathrm{GHz}$ was used to map the continuum. The three configurations were entirely reprocessed and combined both in continuum and ${ }^{12} \mathrm{CO}$ to improve the signal-to-noise ratio $(\mathrm{S} / \mathrm{N})$ and the quality of the data as well as to greatly reduce the residual sidelobes in the final cleaned images. Data calibration was performed using the Common Astronomy Software Application (CASA, see McMullin et al. 2007) version 4.5.3 for Cycle 3 observations and 5.1.1 for the Cycle 5 observations. Flux calibrators were manually checked and the flux calibration uncertainty was estimated to be $20 \%$. For each individual exposure, phase self-calibration was computed on the high signal-to-noise continuum and applied to both the continuum and ${ }^{12} \mathrm{CO}$ data. The Cycle 5 continuum peak (centered at $\left.\alpha_{\mathrm{J} 2000}: 04^{\mathrm{h}} 27^{\mathrm{m}} 02 \mathrm{~s} 573, \delta_{\mathrm{J} 2000}:+26^{\circ} 05^{\prime} 30^{\prime \prime} \cdot 170\right)$ was defined as a phase reference. Relative phase shifts between all different exposures from the three configurations were automatically corrected by the self-calibration procedure. Final calibrated data sets from the three different configurations were combined with their default weights. Before combination, we checked that the average weights of each data set were similar. Joint deconvolution was achieved by the CASA tclean procedure using the Hogbom algorithm and Briggs weighting scheme with a robust parameter of -1 for the continuum data and 0.5 for the CO. The resulting continuum emission map has a synthesized beam of $0.07^{\prime \prime} \times 0.11^{\prime \prime}$ with a root mean square (rms) noise of $50 \mu \mathrm{Jy}$ beam $^{-1}$. The final ${ }^{12} \mathrm{CO}(2-1)$ datacube has a synthesized beam of $0.13^{\prime \prime} \times 0.18^{\prime \prime}$ with an rms noise of $2 \mathrm{mJy} \mathrm{beam}^{-1}$ per $0.3 \mathrm{~km} \mathrm{~s}^{-1}$ spectral channel. For the more compact ${ }^{13} \mathrm{CO}(2-1)$ and $\mathrm{C}^{18} \mathrm{O}(2-1)$ emission, we used the pipeline reduced datacubes from the compact $\mathrm{C} 36-2 / 3$ and intermediate C40-5 configurations, with resulting beams of $0.97^{\prime \prime} \times 0.78^{\prime \prime}$ and $0.23^{\prime \prime} \times 0.34^{\prime \prime}$, respectively, and rms noise per $0.3 \mathrm{~km} \mathrm{~s}^{-1}$ spectral channel of 6 and $5 \mathrm{mJy}_{\text {beam }}^{-1}$, respectively. Our largest recoverable scale is $11^{\prime \prime}$.

\section{Results and analysis}

\subsection{A large and structured disk}

Figure 1a shows the $230 \mathrm{GHz}$ continuum image. The continuum emission is elliptical and centrally peaked, indicating a dominant contribution from the circumstellar disk. The peak intensity is $26 \mathrm{mJy} \mathrm{beam}^{-1}(80 \mathrm{~K})$ and the integrated intensity is $430 \pm$ $30 \mathrm{mJy}$. The difference with Zapata et al. (2015) (590 $\pm 30 \mathrm{mJy})$ and Guilloteau et al. (2011) (531 mJy) is within the calibration uncertainties of $20 \%$. We fit an elliptical Gaussian with the CASA function IMFIT to derive a position axis (PA) of $25.7 \pm 0.3^{\circ}$ for the disk major axis, which is consistent with the previous determinations of $24 \pm 1^{\circ}$ by Guilloteau et al. (2011) and $30 \pm 5^{\circ}$ by Zapata et al. (2015). Assuming that the disk is axisymetric and that the millimetric continuum emission is mainly located in the midplane, we also derived the disk axis inclination to the line of sight at $i=63^{\circ} \pm 2^{\circ}$, which is consistent with the estimate of $64^{\circ} \pm 2^{\circ}$ by Guilloteau et al. (2011).

Figure $1 \mathrm{~b}$ shows the intensity profile of the continuum along the disk major axis. The effective radius encompassing $95 \%$ of the deprojected continuum flux distribution $\int I_{v}(r) 2 \pi r \mathrm{~d} r$ is $R_{\text {eff }, 95 \%}=1.24^{\prime \prime}=174 \mathrm{au}$. The profile shows a centrally peaked component with two symmetrical emission bumps. The deprojected and azimuthally averaged radial profile (dashed red line 
Table 1. Best fit parameters to the disk continuum emission profile.

\begin{tabular}{|c|c|c|c|c|c|c|c|c|c|}
\hline \multicolumn{4}{|c|}{ Main profile } & \multicolumn{3}{|c|}{ Inner ring } & \multicolumn{3}{|c|}{ Outer ring } \\
\hline $\begin{array}{l}\text { A } \\
\left(\mathrm{mJy} \mathrm{beam}^{-1}\right)\end{array}$ & $\begin{array}{c}r_{t} \\
(\mathrm{AU})\end{array}$ & $\gamma$ & $\beta$ & $\begin{array}{c}B_{1} \\
\left(\mathrm{mJy} \mathrm{beam}^{-1}\right)\end{array}$ & $\begin{array}{c}R_{1} \\
(\mathrm{AU})\end{array}$ & $\begin{array}{c}\sigma_{1} \\
(\mathrm{AU})\end{array}$ & $\begin{array}{c}B_{2} \\
\left(\mathrm{mJy} \mathrm{beam}^{-1}\right)\end{array}$ & $\begin{array}{c}R_{2} \\
(\mathrm{AU})\end{array}$ & $\begin{array}{c}\sigma_{2} \\
(\mathrm{AU}) \\
\end{array}$ \\
\hline $30 \pm 1$ & $18 \pm 1$ & $0.07 \pm 0.02$ & $0.7 \pm 0.2$ & $1.5 \pm 0.1$ & $62.3 \pm 0.5$ & $4.7 \pm 0.5$ & $0.4 \pm 0.02$ & $135 \pm 1$ & $22 \pm 1$ \\
\hline
\end{tabular}

in Fig. 1b) shows that this feature is axisymmetric, albeit with a lower contrast due to the large inclination of the system. We follow the modeling approach developed by Long et al. (2018) for similar intensity profiles in Class II disks. We fit the intensity profile with an exponentially tapered power-law for the central component and two Gaussian components for the emission bumps (see Appendix A for details). The best fit components are shown in Fig. 1b together with the fit residuals; the parameters are given in Table 1 . The derived model radial intensity profile (before beam convolution) is shown in Fig. A.1. The possible origins of the observed structures are discussed in Sect. 4.1.

Figures B. 1 and B.2 present channel maps in ${ }^{13} \mathrm{CO}$ and $\mathrm{C}^{18} \mathrm{O}$. They exhibit the characteristic "double loop" pattern and trace the upper and lower surfaces of disks in Keplerian rotation, as in IM Lupi (Pinte et al. 2018), for example. The $\mathrm{C}^{18} \mathrm{O}$ emitting layers appear to be at $z / r \simeq 0.3$, which is similar to IM Lupi. A contribution from the redshifted outflow is also observed in ${ }^{13} \mathrm{CO}$ at $\delta z<5^{\prime \prime}$ and for $\left(V-V_{\text {sys }}\right) \leq 2.5 \mathrm{~km} \mathrm{~s}^{-1}$ (see Fig. B.1). Figure 1c shows the ${ }^{13} \mathrm{CO}$ position-velocity $(\mathrm{PV})$ diagram perpendicular to the flow axis, averaged over $-2^{\prime \prime}<\delta z<2^{\prime \prime}$ to increase the $\mathrm{S} / \mathrm{N}$. We also show maximal projected velocities expected for a disk in Keplerian rotation as well as a rotating infalling shell model (see Sect. 3.2.2 for more details on this model). The kinematics are clearly best fit by Keplerian rotation out to $r=5^{\prime \prime}$. However, faint redshifted emission is also seen at $-2^{\prime \prime}<\delta x<0$ (the wrong direction for rotation), requiring residual infall on the front side of the disk (see green curve in Fig. 1c). The fact that we do not see a symmetric infall signature at negative velocities and $\delta x>0$ may come from asymetrical infall as seen, for example, in L1489-IRS by Yen et al. (2014). We thus concentrate on the blue wing of the PV diagram, which is less affected by outflow contributions. It shows a clear Keplerian profile out to $r=5^{\prime \prime}$, corresponding to a disk radius of $R_{\mathrm{CO}}=700 \mathrm{au}$. The same disk size is indicated in ${ }^{12} \mathrm{CO}$ (Fig. 2). We estimated the stellar mass by using the model of a thin disk in Keplerian rotation. With an inclination $i=63^{\circ}$, the best fit to the $3 \sigma$ contour of the ${ }^{13} \mathrm{CO}$ PV diagram is found for a systemic local standard of rest (LSR) velocity $V_{\text {sys }}=6.35 \pm 0.05 \mathrm{~km} \mathrm{~s}^{-1}$ and a stellar mass $M_{\star}=1.1 \pm 0.2 M_{\odot}$. This is the most accurate determination so far of the protostellar mass for DG Tau B.

\section{2. ${ }^{12} \mathrm{CO}$ redshifted lobe: Nested rotating outflows}

Figure 2 illustrates the change in morphology of the CO redshifted lobe emission as a function of velocity. Individual channel maps are shown in Fig. B.3. At large velocities $\left(V-V_{\text {sys }}=\right.$ $2-8 \mathrm{~km} \mathrm{~s}^{-1}$ ), channel maps show a striking limb-brightened conical morphology with an almost constant opening angle out to at least $z=10^{\prime \prime}=1400 \mathrm{au}$. At lower velocities $\left(V-V_{\text {sys }}=\right.$ $1.2-1.8 \mathrm{~km} \mathrm{~s}^{-1}$ ), a wider, thicker, nonconical component dominates the emission. The transition in morphology between low and mid-high velocities can also be observed on the transverse PV diagrams in Fig. 3 where the slope of the velocity gradient steepens at around $V-V_{\text {sys }} \simeq 2-2.5 \mathrm{~km} \mathrm{~s}^{-1}$. Although it is not

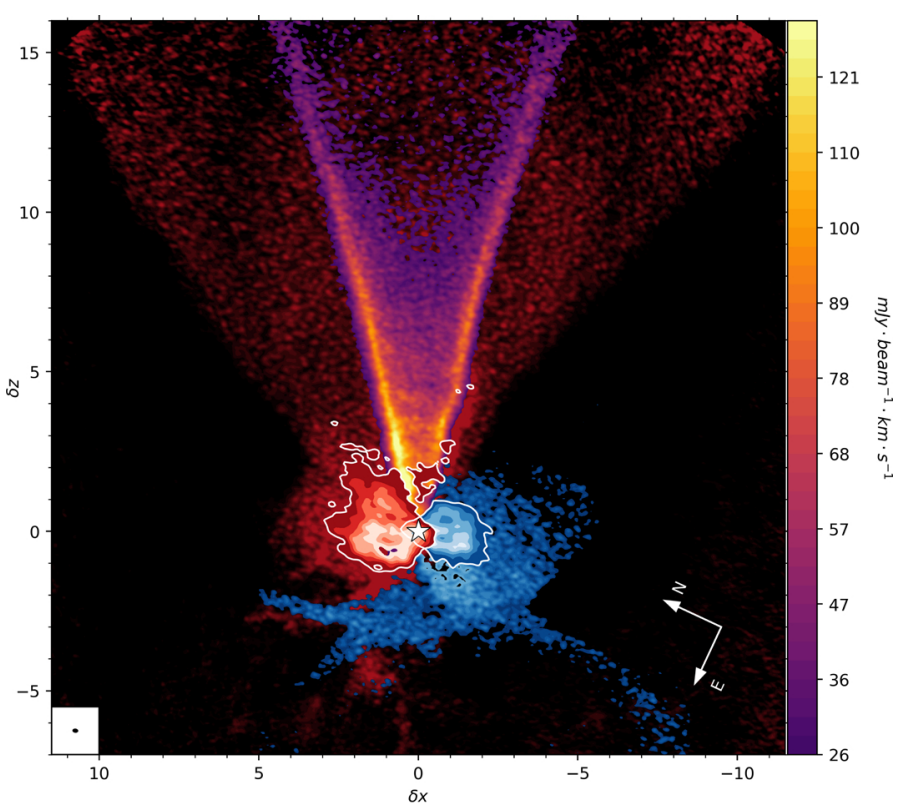

Fig. 2. Summary of the various ${ }^{12} \mathrm{CO}(2-1)$ kinematical components in DG Tau B: the inner conical flow (integrated from $(V-$ $V_{\text {sys }}$ ) $=+2.15$ to $+8 \mathrm{~km} \mathrm{~s}^{-1}$ ) is shown in purple to yellow shades (floor at $\left.26 \mathrm{mJy}_{\text {beam }}{ }^{-1} \mathrm{~km} \mathrm{~s}^{-1}\right)$. The individual low-velocity ${ }^{12} \mathrm{CO}(2-1)$ channel maps at $\left(V-V_{\text {sys }}\right)=1.19 \mathrm{~km} \mathrm{~s}^{-1}$ (resp. $\left.-1.03 \mathrm{~km} \mathrm{~s}^{-1}\right)$ are shown in red (resp. blue) shades (floor at $3 \sigma$ with $\sigma=2 \mathrm{mJy} \mathrm{beam}^{-1}$ ). Inside the white contours, the ${ }^{13} \mathrm{CO}(2-1)$ channel maps are shown at $\left(V-V_{\text {sys }}\right)=$ $1.45 \mathrm{~km} \mathrm{~s}^{-1}$ in red and $\left(V-V_{\text {sys }}\right)=-1.55 \mathrm{~km} \mathrm{~s}^{-1}$ in blue, highlighting the upper and lower disk surfaces (floor at $3 \sigma$ with $\sigma=5 \mathrm{mJy} \mathrm{beam}^{-1}$ ).

clear whether these two kinematical components trace two physically distinct mechanisms, we discuss their specific properties separately below.

\subsubsection{Inner rotating conical flow at $\left(V-V_{\text {sys }}\right)>2 \mathrm{~km} \mathrm{~s}^{-1}$}

The channel maps presented in Fig. 3 illustrate the conical morphology of the emission at $\delta z \leq 10^{\prime \prime}$ and at $\left(V-V_{\text {sys }}\right)=2$ and $5 \mathrm{~km} \mathrm{~s}^{-1}$ and they show a decreasing opening angle with an increasing line-of-sight velocity. The outer cone is defined from the channel map at $2.15 \mathrm{~km} \mathrm{~s}^{-1}$, which corresponds to the lowest velocity channel where the conical component dominates the emission until $\delta z \approx 10^{\prime \prime}$. The derived outer semi-opening angle of $\simeq 18^{\circ}$ strikingly coincides with the opening of the nearinfrared (NIR) scattered light cavity observed by Padgett et al. (1999). Figure 3 shows that the cone opening angle decreases to $12^{\circ}$ at $\left(V-V_{\text {sys }}\right)=5.0 \mathrm{~km} \mathrm{~s}^{-1}$, and Fig. B. 3 shows that it stays constant at higher velocities. This strong velocity stratification and hollow cone geometry is confirmed by the shape of the transverse PV diagrams, which were constructed by positioning a pseudo-slit perpendicular to the flow axis (right panels of Fig. 3). The outer contours of the PV diagrams decrease in 

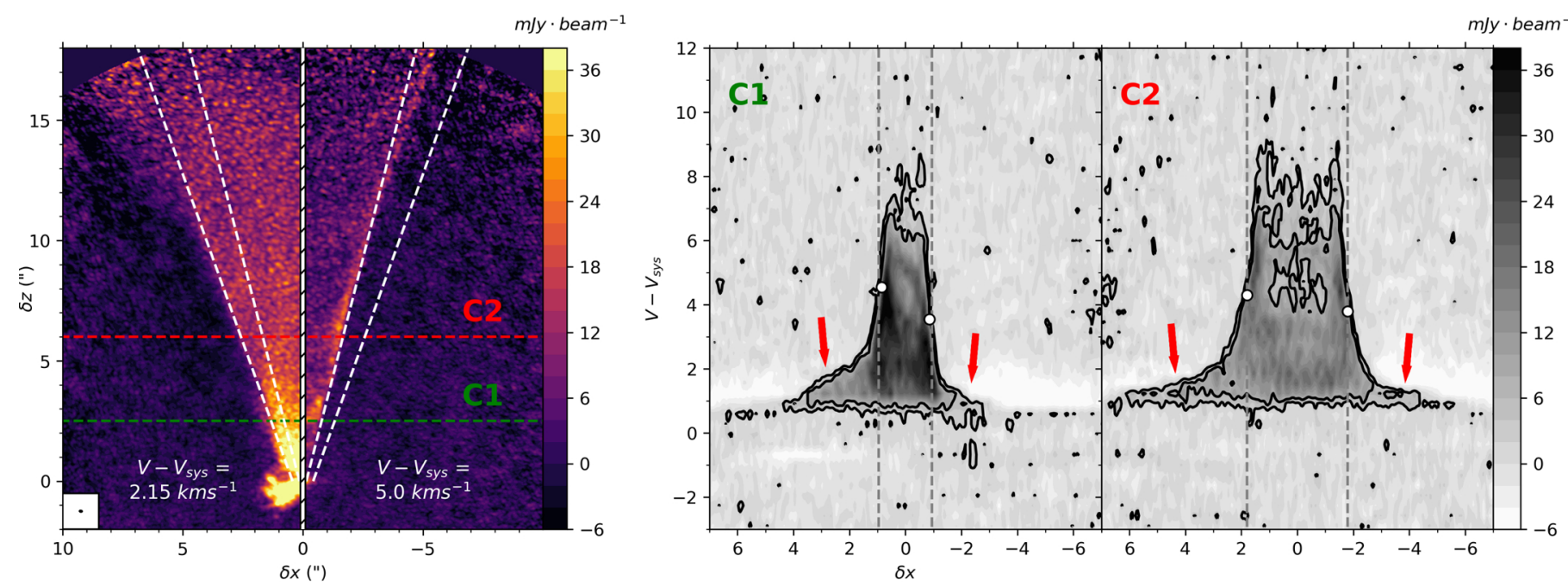

Fig. 3. Left panel: ${ }^{12} \mathrm{CO}$ channel maps at $\left(V-V_{\text {sys }}\right)=2.15 \mathrm{~km} \mathrm{~s}^{-1}$ and $\left(V-V_{\text {sys }}\right)=5.00 \mathrm{~km} \mathrm{~s}^{-1}$. The two white dashed lines trace the outer and inner cones (see text). Only one side of the outflow is shown in each of the two channel maps. The color scale is the same for the two channel maps. Right panel: transverse PV diagrams at $\delta z=2.5^{\prime \prime}$ and $\delta z=6^{\prime \prime}$ obtained along the $\mathrm{C} 1$ and $\mathrm{C} 2$ cuts shown on the channel maps and averaged on a slice of $\Delta z=0.2^{\prime \prime}$. The black contours trace the $2 \sigma$ and $5 \sigma$ levels. The two white points represent the symmetric edges of the PV diagrams at $\delta x= \pm 0.85^{\prime \prime}$ and $\delta x= \pm 1.8^{\prime \prime}$, respectively. Their velocity difference gives an estimate of the rotation velocity (see text).

radii with increasing projected velocities, and then they become almost vertical at the highest velocities $\left(V-V_{\text {sys }}\right) \geq 5 \mathrm{~km} \mathrm{~s}^{-1}$, defining a limiting inner cone. Because the velocity gradient in the conical component is limited to a rather thin shell and narrow range of angles, this suggests a "shear-like" velocity structure within the cone walls with faster material on the internal side.

By extrapolating the conical morphology fit by the naked eye to the channel maps of Fig. 3 at $\delta z<10^{\prime \prime}$, we derived upper limits to the anchoring radii in the range of 10 to $60 \mathrm{au}$ for the inner and outer cone, respectively. No important dynamical or structural changes are observed along the outflow cone over its extent, except for a small increase in the opening angle at $\delta z>$ $10^{\prime \prime}$, suggesting a quasi steady-state flow.

The PV cuts also reveal a systematic velocity difference between symmetric positions at $\pm r$ from the flow axis, especially at low heights above the disk (see white circles in Fig. 3). Assuming axi-symmetry, this difference can only be due to rotation. We thus confirm the finding of Zapata et al. (2015) that the conical redshifted outflow component rotates coherently with altitude and in the same direction as the disk. We observe a velocity shift $\Delta V=1.0 \mathrm{~km} \mathrm{~s}^{-1}$ at $z=2.5^{\prime \prime}$ at a radius of $r=0.85^{\prime \prime}(=120 \mathrm{au})$, indicating a typical specific angular momentum of $\simeq 65$ au km s${ }^{-1}$ for $V_{Z} \approx 9 \mathrm{~km} \mathrm{~s}^{-1}$. A similar value is found at other positions along the cone (Fig. 3).

Assuming a steady flow, the mass flux along the conical structure is estimated from $\left(V-V_{\text {sys }}\right)=2.15$ to $8 \mathrm{~km} \mathrm{~s}^{-1}$ at $\dot{M}=1.7-2.9 \times 10^{-7} M_{\odot} \mathrm{yr}^{-1}$ depending on the opacity hypothesis (see Appendix $\mathrm{C}$ for more details). The derived mass flux is $35 \pm 10$ times larger than the mass flux of the receding atomic jet of $6.4 \times 10^{-9} M_{\odot} \mathrm{yr}^{-1}$ estimated by Podio et al. (2011), making it of the same order as the mass accretion rate onto the star if a standard ejection/accretion ratio of 0.01-0.1 (Hartigan et al. 1994) is adopted for the atomic jet. On the other hand, the linear momentum flux for the inner $\mathrm{CO}$ conical outflow is on the same order of magnitude as that of the jet (two to four times higher depending on the opacity hypothesis). Similar results were found in the smaller conical outflow of HH30 (Louvet et al. 2018) and in the inner molecular outflow shells of the Class 0/I source HH46-47 (Zhang et al. 2019).

\subsubsection{Outer low-velocity wide-angle outflow}

Our ALMA maps reveal that the inner conical outflow is nested inside a wider component $\left(R \approx 10^{\prime \prime}\right.$ at $\left.Z=15^{\prime \prime}\right)$ observed at low redshifted velocities $\left(V-V_{\text {sys }}=1-2 \mathrm{~km} \mathrm{~s}^{-1}\right)$. This structure is shown in Figs. 2 and 4 and can also be seen on transverse PV diagrams in the form of an extended pedestal with a shallower $V(r)$ slope at $\left(V-V_{\text {sys }}\right) \leq 2 \mathrm{~km} \mathrm{~s}^{-1}$ (red arrows on Fig. 3). An important part of the ${ }^{12} \mathrm{CO}$ emission from this component falls at velocities $\left(V-V_{\text {sys }}\right) \leq 1 \mathrm{~km} \mathrm{~s}^{-1}$ where it is absorbed by the surrounding medium or resolved out by ALMA. Contrary to the conical outflow, the wide and slow component shows a parabolic morphology that shifts away from the source along the outflow axis as the line-of-sight velocity increases for $\left(V-V_{\text {sys }}\right)=1.2$ to $1.8 \mathrm{~km} \mathrm{~s}^{-1}$ (see Fig. 4). Discrete loops are also observed in the channel maps, suggesting time variability and contributions from different layers inside this region. A strong transverse asymmetry of the redshifted emission can be observed, for instance, at $\left(V-V_{\text {sys }}\right)=+1.2 \mathrm{~km} \mathrm{~s}^{-1}$, namely; the emission at a given height is more extended toward $\delta x>0$ than $\delta x<0$. Transverse PV cuts in Fig. 3 show that this is due to a global "skew" across the low-velocity pedestal, in the sense that emission at positive $\delta x$ is shifted slightly further into the red compared to symmetric negative $\delta x$ where it is strongly absorbed. This skew is indicative of rotation in the same direction as the disk (and the inner conical flow), but because of absorption by the ambient cloud, it cannot be easily quantified.

In Fig. 4, we compare the morphology and kinematics of this wide-angle component with analytical predictions for an axisymetric rotating shell falling ballistically onto the northwest surface of the disk around a central point mass of $1.1 M_{\odot}$ using the dynamics and structure computed by Ulrich (1976) (see details in Appendix D). Although the model is idealized, it allows one to visualize the key $3 \mathrm{D}$ projection effects expected at $i=63^{\circ}$ in such a geometry. The predicted locus of the shell in each channel map is shown as white contours, for a centrifugal radius $R_{\mathrm{d}}=700$ au and an initial polar angle from the disk axis $\theta_{0}=70^{\circ}$. The effect of varying $R_{\mathrm{d}}$ and $\theta_{0}$ is illustrated in Fig. D.1. No combination of infall parameters $R_{\mathrm{d}}$ and $\theta_{0}$ can reproduce both the 

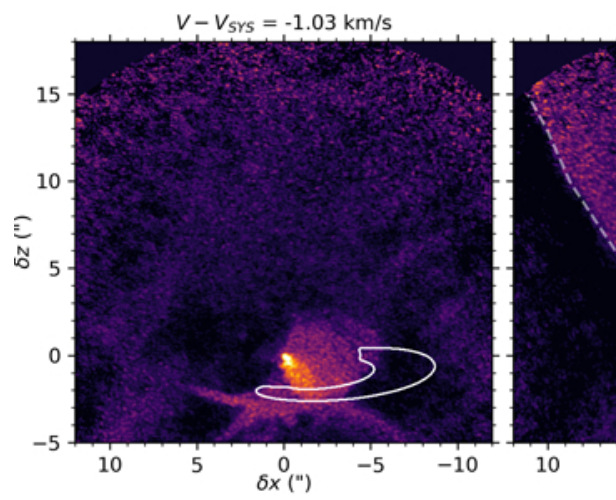

$V-V_{\text {SYS }}=1.19 \mathrm{~km} / \mathrm{s}$

$V-V_{\text {SYS }}=1.51 \mathrm{~km} / \mathrm{s}$

$V-V_{S Y S}=1.83 \mathrm{~km} / \mathrm{s} \quad \mathrm{m} / \mathrm{y} \cdot \mathrm{beam}^{-1}$

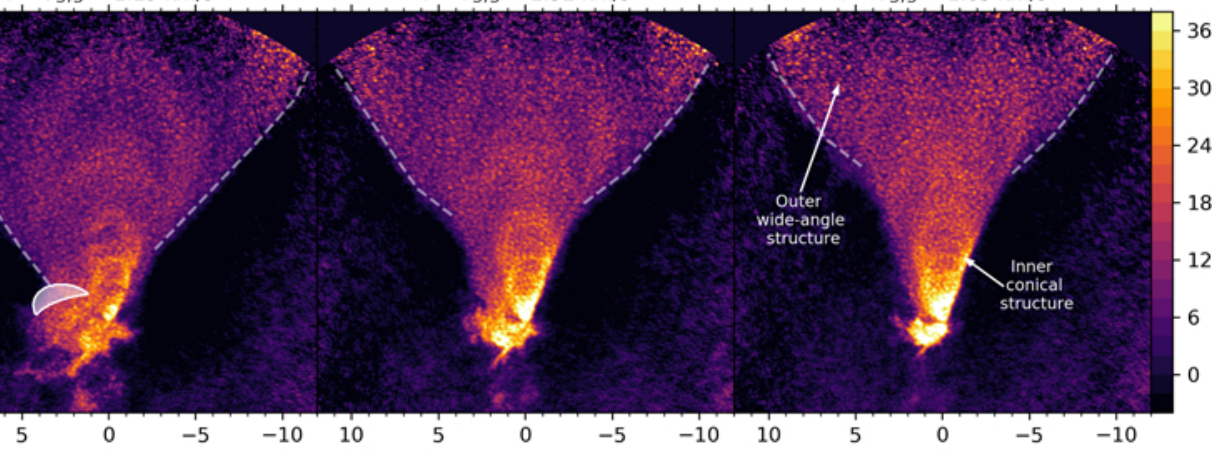

Fig. 4. ${ }^{12} \mathrm{CO}$ individual channel maps at different line-of-sight velocities. The gray filled white contours trace the model of an infalling shell with $r_{\mathrm{d}}=700 \mathrm{au}$ and $\theta_{0}=70^{\circ}$. The contours are not filled when the emission is arising from the back side of the infalling shell. The contour of the slow wide-angle component is indicated by the white dashed line. The color scale is the same for all the channel maps.

large extent and the apparent acceleration of the wide redshifted component. The match would be even worse if infall is slown down by pressure gradients, as in the Class I source TMC1A (Aso et al. 2015). The only feature indicative of infall in the receding lobe of the system is the blueshifted arc opening toward the northwest from $z \simeq-2.2^{\prime \prime}$, which is suggestive of the back side of an infalling shell from $\theta_{0}>70^{\circ}$ (see leftmost panel of Fig. 4). Therefore, we conclude that residual infall in the receding lobe is confined to equatorial regions, and the wide angle redshifted component surrounding the inner cone traces mostly outflowing material.

\section{Discussion}

\subsection{DG Tau B disk: Evolutionary stage and origin of structure}

DG Tau B has been traditionally classified as a Class I source based on the slope of its near-infrared spectral energy distribution (Luhman et al. 2010). However, Crapsi et al. (2008) showed that strongly inclined Class II disks $\left(i \geq 65^{\circ}\right)$ could be misclassified as Class I with the spectral slope method, and Kruger et al. (2011) attribute ice absorptions toward DG Tau B to a thick edge-on disk. Our ALMA observations show that while DG Tau B's disk is indeed very large and flared, residual infall onto the disk is still present at large polar angles $\theta_{0}>70^{\circ}$ in both lobes of the system (see Sects. 3.1 and 3.2.2). However, by extrapolating the single-dish fluxes obtained by Mottram et al. (2017) at $450 \mu \mathrm{m}$ and $850 \mu \mathrm{m}$, we find that the extended dust envelope around DG Tau B contributes only $300_{-300}^{+400} \mathrm{mJy}$ at $1.3 \mathrm{~mm}$, that is, at most comparable to the disk flux itself. Following Eq. (1) of Motte \& André (2001), we would infer an envelope mass $<0.37 M_{\odot}$ within $15^{\prime \prime}(2000 \mathrm{au})$, that is, less than one third the stellar mass. Hence we conclude that DG Tau B is in the late Class I phase, and it is in the final stages of clearing up its infalling envelope.

We note that DG Tau B's disk is as large as the largest known Class II disks. Two other large Class I disks with dust and gas sizes similar to DG Tau B are GY $91\left(R_{\text {dust }}=140 \mathrm{au}\right.$, van der Marel et al. 2019) and L1489-IRS $\left(R_{\text {dust }}=250 \mathrm{au}, R_{\mathrm{CO}}=700 \mathrm{au}\right.$, Yen et al. 2014). Hence, the trend of increasing gas disk size with age reported by Najita \& Bergin (2018) may be affected by low-number statistics, and an unbiased survey of Class I disks appears necessary to investigate disk size evolution at $\leq 1$ Myr.

DG tau B is the third Class I disk where axisymmetric bumps of enhanced emission were reported, after TMC1-A (Bjerkeli et al. 2016; Harsono et al. 2018) and GY 91 (Sheehan \& Eisner 2018). All three disks show bumps and dips with low contrast compared to the main emission profile.

In the model continuum intensity profile of DG Tau B, we see a depression preceding the first emission bump (Fig. A.1) that might trace the possible signature of a gap. Following the method outlined in Long et al. (2018), we estimate a gap center of $R_{g}=53$ au with a width of $\Delta=9$ au, which is typical of those recently observed in Class II disks (Long et al. 2018; Andrews et al. 2018). Assuming that $\Delta$ is 5.5 times the planet's Hill radius (Lodato et al. 2019), the mass of the planet that could carve the inner gap in DG Tau B would be $0.1 M_{\mathrm{J}}$.

Alternatively, the observed emission bumps in the DG Tau B disk may be due to other mechanisms than rings and gaps carved by planets. Indeed, the radial emission profile does not show dips going below the underlying centrally peaked powerlaw component, which is unlike what has been observed, for example, in the HL Tau disk (Brogan et al. 2015). We might therefore be witnessing an earlier stage of millimetric dust accumulation before planetary and gap formation. Different mechanisms can lead to the formation of radial structures in disks. Dust radial trapping and growth due, for example, to snow lines (Zhang et al. 2015) as well as the secular gravitational instability (Takahashi \& Inutsuka 2016) have been recently invoked to reproduce the rings in the HL Tau disk. This latter mechanism could act in the large and likely massive DG Tau B disk. Nonideal magnetohydrodynamic (MHD) effects and zonal flows can also generate radial structures in disks, with lower contrast at large radii (e.g., in Béthune et al. 2017; Suriano et al. 2019). In any case, our DG Tau B ALMA observations confirm that disk substructures can form at an early stage where infall and outflow are still present and could potentially affect the disk structure and possibly planet formation.

\subsection{Origin of the nested rotating outflows in DG Tau B}

Our ALMA observations reveal that the inner bright conical CO outflow in DG Tau B is surrounded by outflowing material. Therefore, it is not tracing the interface between infall and outflow. This interface must be located at much larger angles ( $\geq 70^{\circ}$ from the geometry of the lowest velocity shells observed in our channel maps, compared with infall models, see Figs. 3 and 4). An important implication of these results is that outflowing material can occupy a wider solid angle than scattered light cavities suggest. This may explain why classical disk plus envelope models have difficulties in reproducing the 
spectral energy distribution and scattered light cavities images in DG Tau B at the same time (Gramajo et al. 2010) as well as why some Class II sources with active outflows appear to have dusty conical "envelopes" above their disks (e.g., RY Tau, Fig. 3 in Takami et al. 2013.)

The parabolic morphology of the slow wide angle flow and its multiple inner loops resemble the structure of slow outflow cavities around younger sources, such as HH46-47 (Zhang et al. 2019). They are interpreted as ambient material swept up by an episodic wide-angle inner wind. Wind-driven shell models predict emission maps shifting away from the star as the lineof-sight velocity increases as observed in this component (see Fig. 24 in Lee et al. 2000).

However, the striking conical inner flow anchored at small radii $\leq 10-60$ au highly unusual so far. It is reminiscent of the predictions for the long-term interaction shell between inner jet bowshocks and a steady outer disk wind, as recently computed by Tabone et al. (2018). The interaction between an outer disk wind and an inner wide-angle wind cannot be excluded either, but predictions for this scenario do not exist yet.

Alternatively, the constant velocity and mass flux of the inner conical flow, also seen in HH30 (Louvet et al. 2018), suggest that it could trace matter directly ejected from the disk. In the case of a cold, steady, axisymmetric MHD disk wind, our derived value of angular momentum and poloidal speed would predict an ejection radius of $r_{0} \approx 2$ au and a magnetic level arm of $\lambda=1.6$, using the formulae in Anderson et al. (2003). This model was ruled out by Zapata et al. (2015) based on the small magnetic lever arm $\lambda \simeq 2$ and large outflow mass-flux. However, recent nonideal MHD simulations of magnetized winds from protoplanetary disks do predict small magnetic lever arms and a mass-flux of the same order as the accretion rate (Béthune et al. 2017; Bai et al. 2016), as observed here. They also predict an important role of thermal gradients in driving the wind. In that case, the launching radius $r_{0}$ could be up to 5 au (corresponding to the minimum $\lambda \approx 1$ ).

However, we stress that our estimate of angular momentum is highly uncertain given the complex steep velocity gradients, projection effects, and beam smearing effects. Threedimensional modeling of the observations is requested to constrain the flow rotation and kinematic structure more accurately as well as to firmly distinguish between the various scenarios. This is outside the scope of the present Letter and will be the subject of a forthcoming paper (de Valon et al., in prep.).

Whatever its origin, we stress that the large mass-flux carried along the inner conical outflow walls, being comparable to the inner disk accretion rate, must play a major role in limiting the final mass of the star and disk. Thanks to its large disk, proximity, and favorable inclination (compared e.g., to HH30), DG Tau B is a very promising target to elucidate the diskoutflow connexion and its impact on disk evolution and planet formation.

Acknowledgements. The authors would like to thank the referee, whose comments helped improve the quality of the Letter. We acknowledge Ana Lopez Sepulcre for crucial help in the data reduction process at the IRAM-Grenoble ALMA ARC node. This Letter makes use of the following ALMA data: ADS/JAO.ALMA\#2015.1.01108.S, ADS/JAO.ALMA\#2017.1.01605.S. ALMA is a partnership of ESO (representing its member states), NSF (USA) and NINS (Japan), together with NRC (Canada), MOST and ASIAA (Taiwan), and KASI (Republic of Korea), in cooperation with the Republic of Chile. The Joint ALMA Observatory is operated by ESO, AUI/NRAO and NAOJ. Part of the data reduction presented in this Letter were performed on the GRICAD infrastructure (https://gricad.univ-grenoble-alpes.fr), which is partly supported by the Equipe@Meso project (reference ANR-10-EQPX-29-01) of the programme Investissements d'Avenir supervised by the Agence Nationale pour la Recherche. This work was supported by the Programme National de Physique Stellaire (PNPS) and the Programme National de Physique et Chimie du Milieu Interstellaire (PCMI) of CNRS/INSU co-funded by CEA and CNES. L. A. Z. acknowledge financial support from DGAPA, UNAM, and CONACyT, México. F. L. acknowledges the support of the Fondecyt program $n^{\circ} 3170360$.

\section{References}

Anderson, J. M., Li, Z.-Y., Krasnopolsky, R., \& Blandford, R. D. 2003, ApJ, 590, L107

Andrews, S. M., Huang, J., Pérez, L. M., et al. 2018, ApJ, 869, L41

Aso, Y., Ohashi, N., Saigo, K., et al. 2015, ApJ, 812, 27

Bai, X.-N., Ye, J., Goodman, J., \& Yuan, F. 2016, ApJ, 818, 152

Béthune, W., Lesur, G., \& Ferreira, J. 2017, A\&A, 600, A75

Bjerkeli, P., van der Wiel, M. H. D., Harsono, D., Ramsey, J. P., \& Jørgensen,

J. K. 2016, Nature, 540, 406

Brogan, C. L., Pérez, L. M., Hunter, T. R., et al. 2015, ApJ, 808, L3

Brown, A. G. A., Vallenari, A., Prusti, T., et al. 2018, A\&A, 616, A1

Crapsi, A., van Dishoeck, E. F., Hogerheijde, M. R., Pontoppidan, K. M., \& Dullemond, C. P. 2008, A\&A, 486, 245

Frank, A., Ray, T. P., Cabrit, S., et al. 2014, Protostars and Planets VI (Tucson: University of Arizona Press), 451

Goldsmith, P. F., Heyer, M., Narayanan, G., et al. 2008, ApJ, 680, 428

Gramajo, L. V., Whitney, B. A., Gómez, M., \& Robitaille, T. P. 2010, AJ, 139, 2504

Guilloteau, S., Dutrey, A., Piétu, V., \& Boehler, Y. 2011, A\&A, 529, A105

Harsono, D., Bjerkeli, P., van der Wiel, M. H. D., et al. 2018, Nat. Astron., 2, 646

Hartigan, P., Morse, J. A., \& Raymond, J. 1994, ApJ, 436, 125

Kruger, A. J., Richter, M. J., Carr, J. S., et al. 2011, ApJ, 729, 145

Lee, C.-F., Mundy, L. G., Reipurth, B., Ostriker, E. C., \& Stone, J. M. 2000, ApJ, 542,925

Lodato, G., Dipierro, G., Ragusa, E., et al. 2019, MNRAS, 486, 453

Long, F., Pinilla, P., Herczeg, G. J., et al. 2018, ApJ, 869, 17

Louvet, F., Dougados, C., Cabrit, S., et al. 2018, A\&A, 618, A120

Luhman, K. L., Allen, P. R., Espaillat, C., Hartmann, L., \& Calvet, N. 2010, ApJS, 186, 111

McMullin, J. P., Waters, B., Schiebel, D., Young, W., \& Golap, K. 2007, Astron. Data Anal. Softw. Syst. XVI, 376, 127

Mitchell, G. F., Sargent, A. I., \& Mannings, V. 1997, ApJ, 483, L127

Motte, F., \& André, P. 2001, A\&A, 365, 440

Mottram, J. C., van Dishoeck, E. F., Kristensen, L. E., et al. 2017, A\&A, 600, A99

Mundt, R., \& Fried, J. W. 1983, ApJ, 274, L83

Mundt, R., Ray, T. P., \& Raga, A. C. 1991, A\&A, 252, 740

Najita, J. R., \& Bergin, E. A. 2018, ApJ, 864, 168

Padgett, D. L., Brandner, W., Stapelfeldt, K. R., et al. 1999, AJ, 117, 1490

Pinte, C., Ménard, F., Duchêne, G., et al. 2018, A\&A, 609, A47

Podio, L., Eislöffel, J., Melnikov, S., Hodapp, K. W., \& Bacciotti, F. 2011, A\&A, 527, A13

Schmalzl, M., Kainulainen, J., Quanz, S. P., et al. 2010, ApJ, 725, 1327

Sheehan, P. D., \& Eisner, J. A. 2018, ApJ, 857, 18

Suriano, S. S., Li, Z.-Y., Krasnopolsky, R., Suzuki, T. K., \& Shang, H. 2019, MNRAS, 484, 107

Tabone, B., Raga, A., Cabrit, S., \& Pineau des Forêts, G. 2018, A\&A, 614, A119 Takahashi, S. Z., \& Inutsuka, S.-I. 2016, AJ, 152, 184

Takami, M., Karr, J. L., Hashimoto, J., et al. 2013, ApJ, 772, 145

Terebey, S., Shu, F. H., \& Cassen, P. 1984, ApJ, 286, 529

Ulrich, R. K. 1976, ApJ, 210, 377

van der Marel, N., Dong, R., di Francesco, J., Williams, J., \& Tobin, J. 2019,

ApJ, 872, 112

van Kempen, T. A., van Dishoeck, E. F., Salter, D. M., et al. 2009, A\&A, 498, 167

Yen, H.-W., Takakuwa, S., Ohashi, N., et al. 2014, ApJ, 793, 1

Zapata, L. A., Lizano, S., Rodríguez, L. F., et al. 2015, ApJ, 798, 131

Zhang, K., Blake, G. A., \& Bergin, E. A. 2015, ApJ, 806, L7

Zhang, Y., Arce, H. G., Mardones, D., et al. 2016, ApJ, 832, 158

Zhang, Y., Higuchi, A. E., Sakai, N., et al. 2018, ApJ, 864, 76

Zhang, Y., Arce, H. G., Mardones, D., et al. 2019, ApJ, 883, 1 


\section{Appendix A: Model of the radial intensity profile}

To model the radial intensity profile of the continuum emission, we used the equation of an exponentially tapered power law plus two off-centered Gaussians to reproduce the bumps:

$I(r)=A\left(\frac{r}{r_{\mathrm{t}}}\right)^{\gamma} \exp \left[-\left(\frac{r}{r_{\mathrm{t}}}\right)^{\beta}\right]+\sum_{i} B_{i} \exp \left[-\frac{\left(r-R_{i}\right)^{2}}{2 \sigma_{i}}\right]$.
To compare effectively with our observations, we convolved this profile with a kernel with a full width at half maximum (FWHM) corresponding to the beam FWHM in that direction. The fitting is achieved using a nonlinear least squares method. The parameters of the best fit with their standard deviation errors are listed in Table 1 and the model intensity profile without convolution is shown in Fig. A.1.

Table A.1. Log of observations.

\begin{tabular}{lcccccccc}
\hline \hline Cycle Configuration & $\begin{array}{c}\text { Number of } \\
\text { sessions }\end{array}$ & $\begin{array}{c}\text { Date } \\
\text { (YYYY-MM-DD) }\end{array}$ & $\begin{array}{c}\text { Number of } \\
\text { antennas }\end{array}$ & $\begin{array}{c}\text { Baseline range } \\
(\mathrm{m})\end{array}$ & $\begin{array}{c}\text { Total on source } \\
\text { integration time }\end{array}$ & $\begin{array}{c}\text { Flux } \\
\text { calibrator }\end{array}$ & $\begin{array}{c}\text { Phase } \\
\text { calibrator }\end{array}$ \\
\hline 3 & $\mathrm{C} 36-2 / 3$ & 1 & $2016-04-23$ & 39 & $15.1-400$ & $0 \mathrm{~h} 48 \mathrm{mn}$ & $\mathrm{J} 0238+1636$ & $\mathrm{~J} 0510+1800$ \\
3 & $\mathrm{C} 40-5$ & $4(2 \mathrm{kept})^{(a)}$ & $2016-08-05 / 15$ & $43 / 38$ & $15.1-1500 / 1600$ & $1 \mathrm{~h} 37 \mathrm{mn}$ & $\mathrm{J} 0510+1800$ & $\mathrm{~J} 0510+1800$ \\
5 & $\mathrm{C} 43-8$ & 3 & $2017-11-16 / 18 / 19$ & $43 / 44 / 46$ & $92.2-8500$ & $2 \mathrm{~h} 06 \mathrm{mn}$ & $\mathrm{J} 0510+1800$ & $\mathrm{~J} 0438+3004$ \\
\hline
\end{tabular}

Notes. ${ }^{(a)}$ Only two sessions were kept due to poor phase calibration.

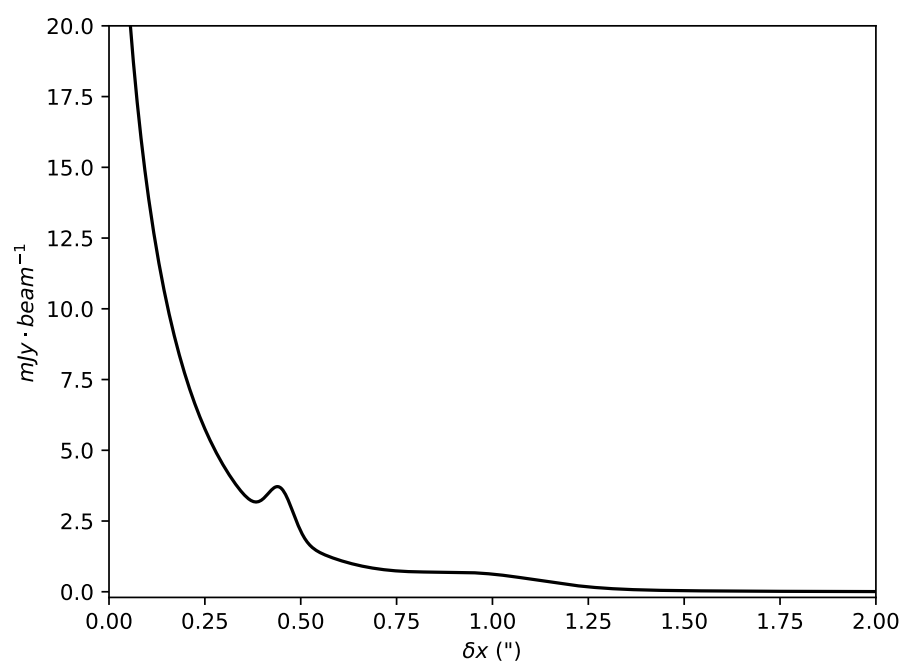

Fig. A.1. Best fit to the radial continuum emission profile before convolution by the beam. 


\section{Appendix B: CO channel maps}

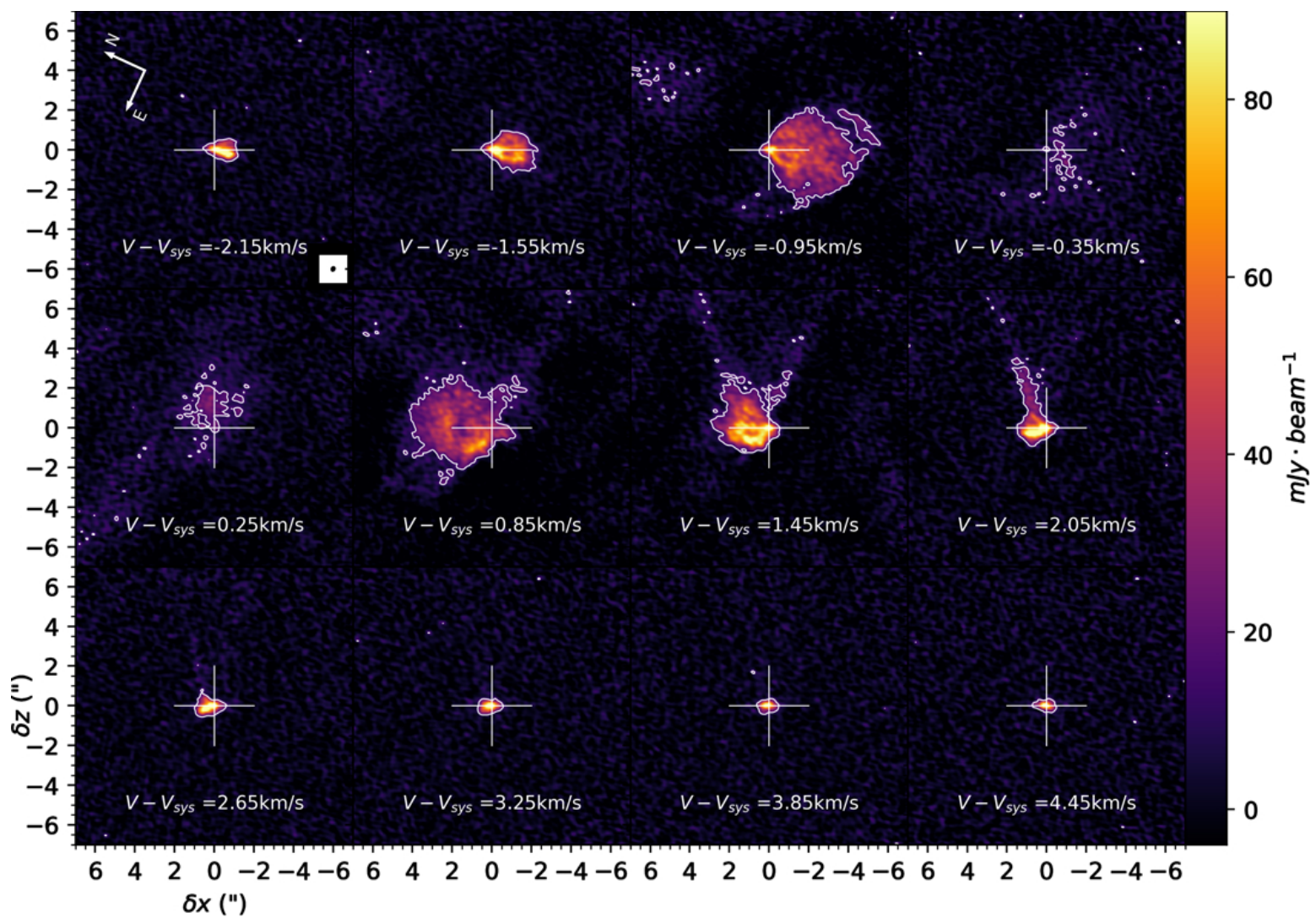

Fig. B.1. ${ }^{13} \mathrm{CO}$ selected individual channel maps. For each channel map $\Delta V=0.3 \mathrm{~km} \mathrm{~s}^{-1}$ and the central velocity is indicated. The white contours trace the $3 \sigma$ limit with $\sigma=5 \mathrm{mJy}_{\text {beam }}{ }^{-1}$ (or $1.6 \mathrm{~K}$ ). The white cross in each panel locates the central position of the continuum. The beam $\left(0.23^{\prime \prime} \times 0.34^{\prime \prime}\right.$ at $\left.\mathrm{PA}=0.5^{\circ}\right)$ is shown in the first panel.

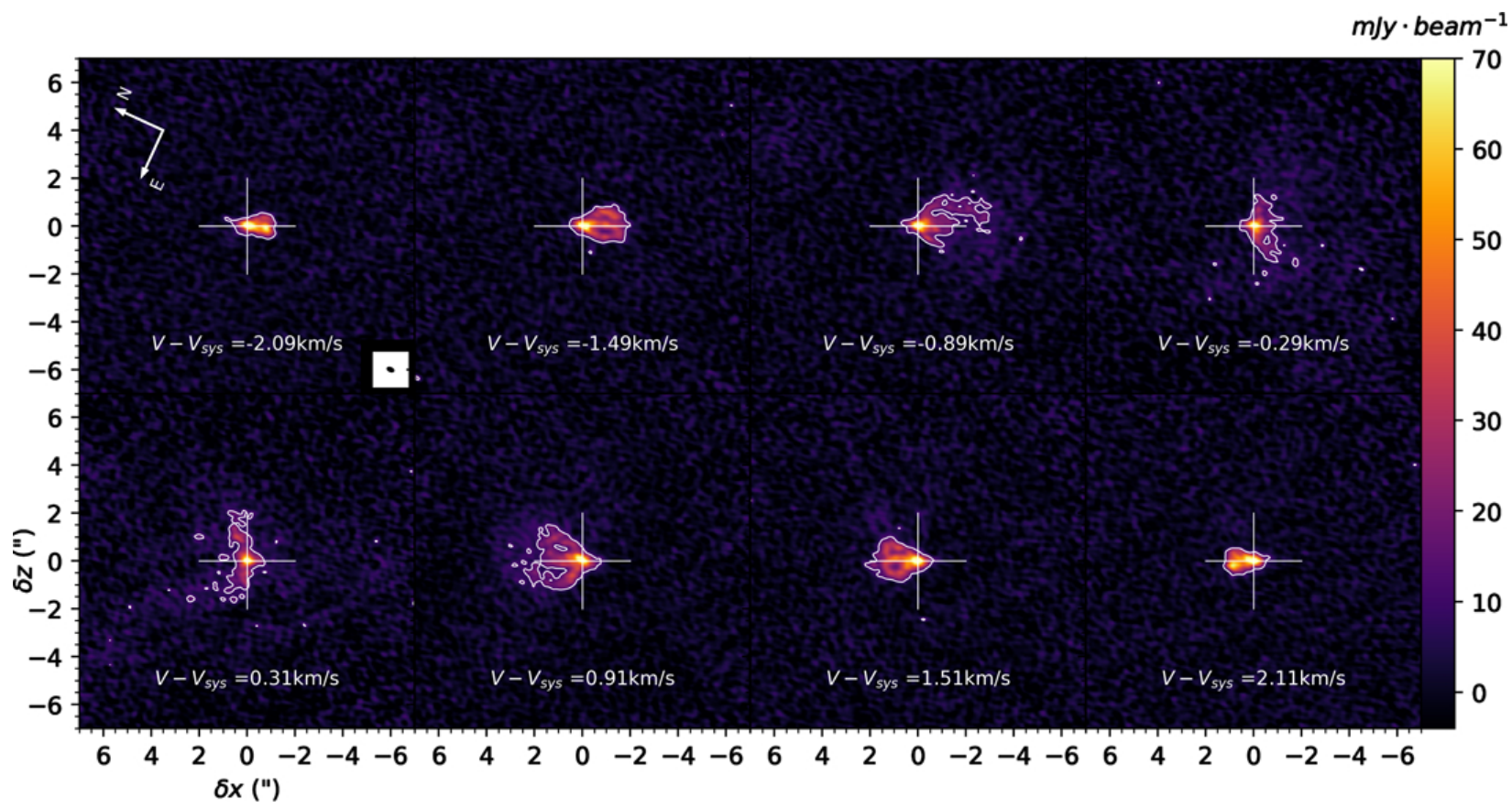

Fig. B.2. $\mathrm{C}^{18} \mathrm{O}$ selected individual channel maps. For each channel map $\Delta V=0.3 \mathrm{~km} \mathrm{~s}^{-1}$ and the central velocity is indicated. The white contours trace the $3 \sigma$ limit with $\sigma=4 \mathrm{mJy}_{\text {beam }}{ }^{-1}$ (or $1.3 \mathrm{~K}$ ). The white cross in each panel locates the central position of the continuum. The beam $\left(0.23^{\prime \prime} \times 0.34^{\prime \prime}\right.$ at $\left.\mathrm{PA}=2^{\circ}\right)$ is shown in the first panel. 


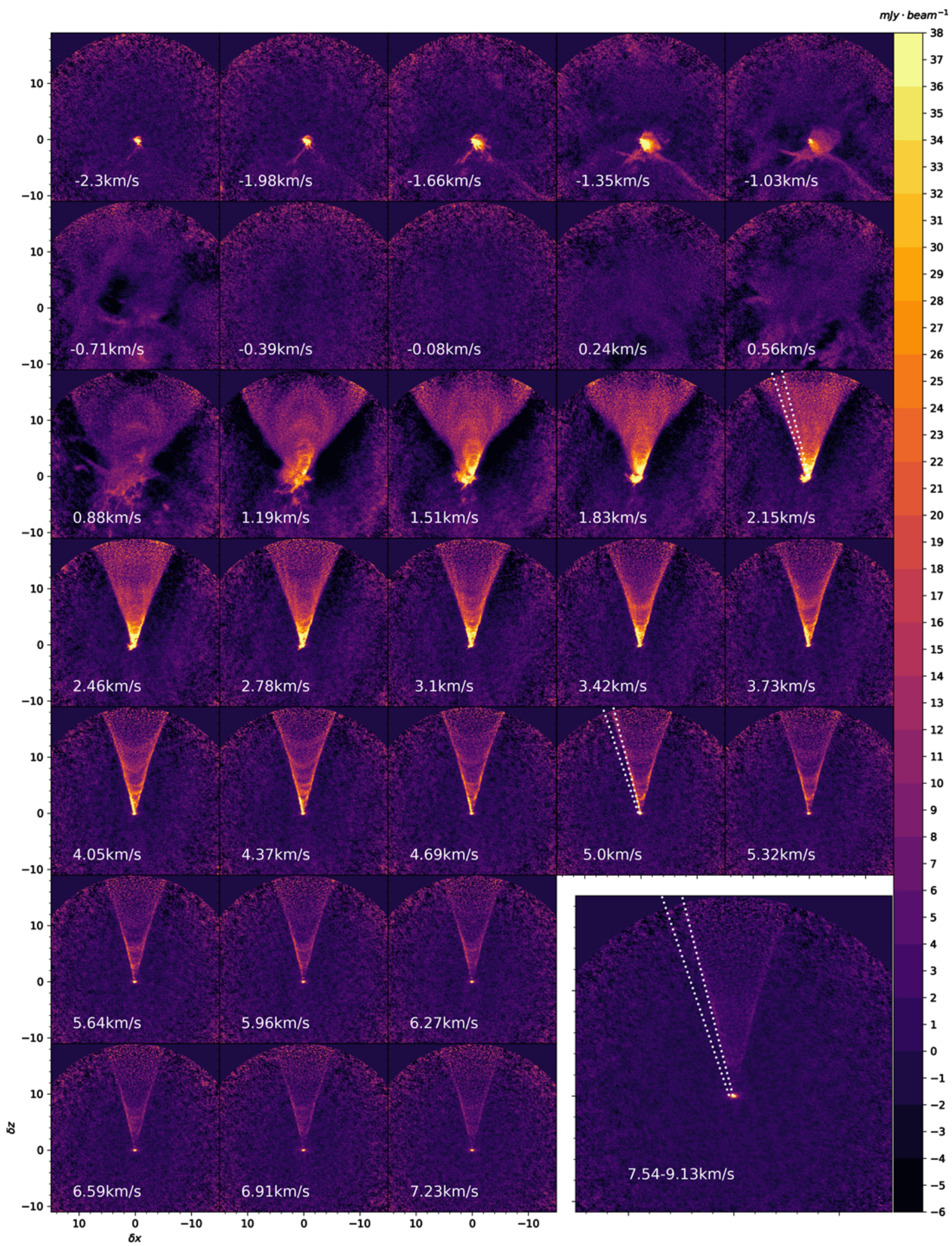

Fig. B.3. ${ }^{12} \mathrm{CO}$ selected individual channel maps. For each channel map $\Delta V=0.32 \mathrm{~km} \mathrm{~s}^{-1}$ and the central velocity is indicated. Bottom right panel: averaged map from $\left(V-V_{\text {sys }}\right)=7.54$ to $\left(V-V_{\text {sys }}\right)=9.13 \mathrm{~km} \mathrm{~s}^{-1}$. The two white dotted lines show the inner and outer cone as defined in Fig. 3 . 


\section{Appendix C: Estimation of the mass flux}
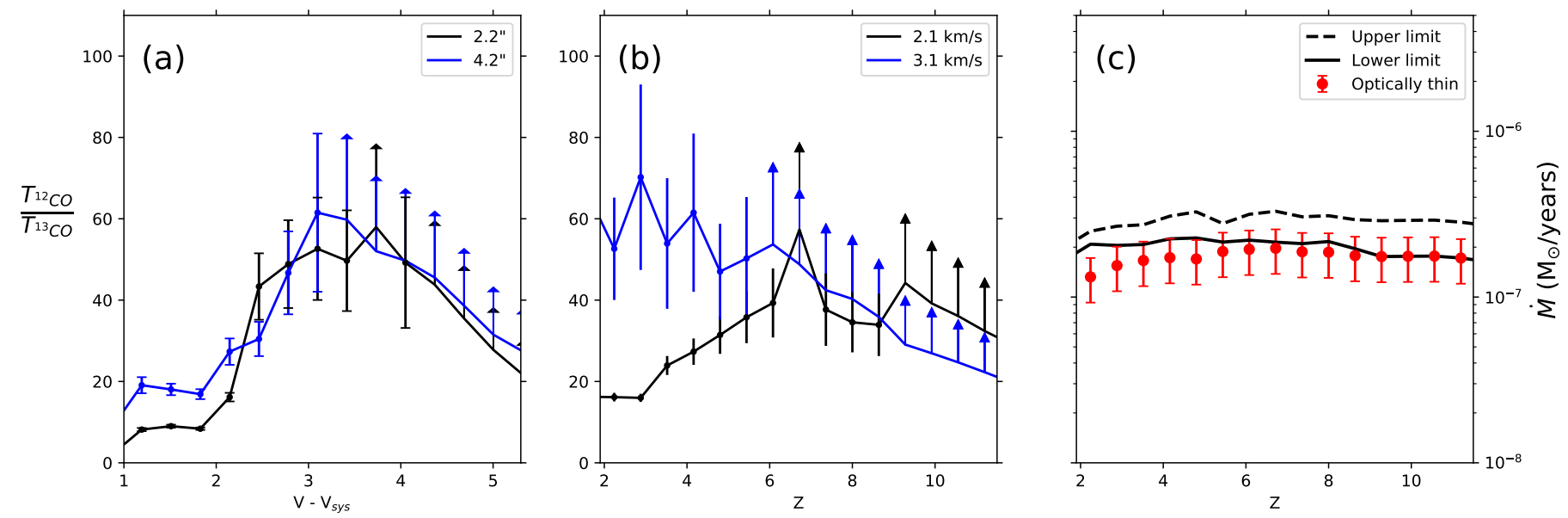

Fig. C.1. Intensity ratio between ${ }^{12} \mathrm{CO}$ and ${ }^{13} \mathrm{CO}$ radially averaged over the cone region: $(a)$ as a function of the velocity on two slices of width $=0.7^{\prime \prime}$ at $\delta z=2.2^{\prime \prime}$ (black) and $\delta z=4.2^{\prime \prime}$ (blue); and $(b)$ as a function of height in the velocity bins $\left(V-V_{\text {sys }}\right)=2.15 \mathrm{~km} \mathrm{~s}^{-1}$ (blue) and $\left(V-V_{\text {sys }}\right)=3.1 \mathrm{~km} \mathrm{~s}^{-1}$ (black). The arrows represent the $5 \sigma$ lower limit on the ratio when no ${ }^{13} \mathrm{CO}$ is detected. (c) Resulting mass flux under different opacity hypothesis assuming optically thin ${ }^{12} \mathrm{CO}$ (in red with errorbars at $30 \%$ ), applying opacity correction based on ${ }^{12} \mathrm{CO} /{ }^{13} \mathrm{CO}$ and assuming either optically thin emission (lower limit: black solid) or the last detected value (upper limit: black dashed) when no ${ }^{13} \mathrm{CO}$ is detected (see text for more details).

We calculated the mass in the conical component assuming LTE excitation and by using the method presented in Louvet et al. (2018). We integrated $T_{\mathrm{mb}}$ from $\left(V-V_{\text {sys }}\right)=2.15$ to $8 \mathrm{~km} \mathrm{~s}^{-1}$ in slices of $\delta z=0.7^{\prime \prime}$ inside the region delimited by the outer cone (see Fig. 3). We chose this velocity range and spatial domain to have a decent estimate of the mass without being too contaminated by the wider component at lower velocities. We set the CO excitation temperature to $T_{\mathrm{ex}}=50 \mathrm{~K}$, corresponding to the peak $T_{\mathrm{mb}}$ in this region. The integrated flux over the cone area in the range $\left(V-V_{\text {sys }}\right)=2.15$ to $8 \mathrm{~km} \mathrm{~s}^{-1}$ and $\delta z=2^{\prime \prime}-10^{\prime \prime}$ is $60 \mathrm{Jy} \mathrm{km} \mathrm{s}^{-1}$, corresponding to a total optically thin mass of $1 \times 10^{-4} M_{\odot}$. A similar integrated flux was found on the SMA data of Zapata et al. (2015) (64 Jy km s${ }^{-1}$ ). For a twice lower $T_{\mathrm{ex}}$, the mass would be decreased by a factor 1.6. The mass scales linearly with $T_{\mathrm{ex}}$ for $T_{\mathrm{ex}} \geq 50 \mathrm{~K}$. If we integrated down to $\left(V-V_{\text {sys }}\right)=1 \mathrm{~km} \mathrm{~s}^{-1}$, encompassing all redshifted lobe emission, the derived mass would typically increase by a factor of 1.5 , but it would suffer from contamination from the wider "pedestal" component projected in front of the cone.

Figures C.1a and $\mathrm{b}$ show that the intensity ratio of ${ }^{12} \mathrm{CO}$ to ${ }^{13} \mathrm{CO}$ increases as a function of the velocity and height in the outflow. We used the compact and more sensitive ${ }^{13} \mathrm{CO}$ configuration for more precision on this variation. We added a standard correction factor for the ${ }^{12} \mathrm{CO}$ optical depth, which was computed for each slice in $\Delta z$ and the velocity bin. The observed ratio is always above 10 so that the ${ }^{13} \mathrm{CO}$ emission is optically thin. The correction factor to be applied to the ${ }^{12} \mathrm{CO}$ brightness is then given by:

$F_{12}=X_{12,13} \frac{T_{13 \mathrm{CO}}}{T_{12 \mathrm{CO}}}$,

with $X_{12,13}$, the abundance ratio set at 65 (Goldsmith et al. 2008). When no ${ }^{13} \mathrm{CO}$ is detected, a lower and upper limit to the mass flux are obtained by assuming optically thin ${ }^{12} \mathrm{CO}$ and by setting the intensity ratio to the upper measured value. The derived lower and upper limits on the mass flux are constant from $\delta z=2^{\prime \prime}$ to $\delta z=12^{\prime \prime}$ at $\dot{M}=1.7-2.9 \times 10^{-7} M_{\odot} \mathrm{yr}^{-1}$ (see Fig. C. $1 \mathrm{c}$ ).

\section{Appendix D: Rotating infall models}

For an axisymmetric rotating envelope in ballistic infall onto a point mass $M$, the equation defining one given streamline is described by only two parameters: $r_{\mathrm{d}}$ the outer disk radius and $\theta_{0}$ the initial infall angle with the rotation axis of the disk (Ulrich 1976). In defining $\theta$, the angle of the streamline with respect to the rotation axis at a given spherical radius $R$, we get:

$\cos \theta=\frac{-r_{\mathrm{d}} \cos \theta_{0} \sin ^{2} \theta_{0}}{R}+\cos \theta_{0}$.

We can then, with $z=r \cos \theta$, describe the shape of the flow surface followed by infalling material originating from $\theta_{0}$. The velocity components at a given point $(z, R)=(\theta, R)$ are given by:

$$
\begin{aligned}
& v_{R}=-\left(\frac{G M}{R}\right)^{\frac{1}{2}}\left(1+\frac{\cos \theta}{\cos \theta_{0}}\right)^{\frac{1}{2}} \\
& v_{\theta}=\left(\frac{G M}{R}\right)^{\frac{1}{2}}\left(\cos \theta_{0}-\cos \theta\right)\left(\frac{\cos \theta_{0}+\cos \theta}{\cos \theta_{0} \sin ^{2} \theta}\right)^{\frac{1}{2}} \\
& v_{\phi}=\left(\frac{G M}{R}\right)^{\frac{1}{2}} \frac{\sin \theta_{0}}{\sin }\left(1-\frac{\cos \theta}{\cos \theta_{0}}\right)^{\frac{1}{2}} .
\end{aligned}
$$

The paper by Ulrich (1976) had a typographical error in the equation of $v_{\theta}$, which was corrected by Terebey et al. (1984) (see their Eq. (89)).

We then consider at each $z(R)$, a ring of streamlines with azimuth $\phi$ from 0 to $2 \pi$. We projected their position onto the plane of the sky and their velocity onto the line of sight, and we convolved in $3 \mathrm{D}$ to produce a synthetic datacube matching the angular and spectral resolutions of our ALMA observations. We show below channel maps predicted by this model for different values of $r_{\mathrm{d}}$ and $\theta_{0}$. 
A. de Valon et al.: ALMA observations of DG Tauri B disk and CO outflow

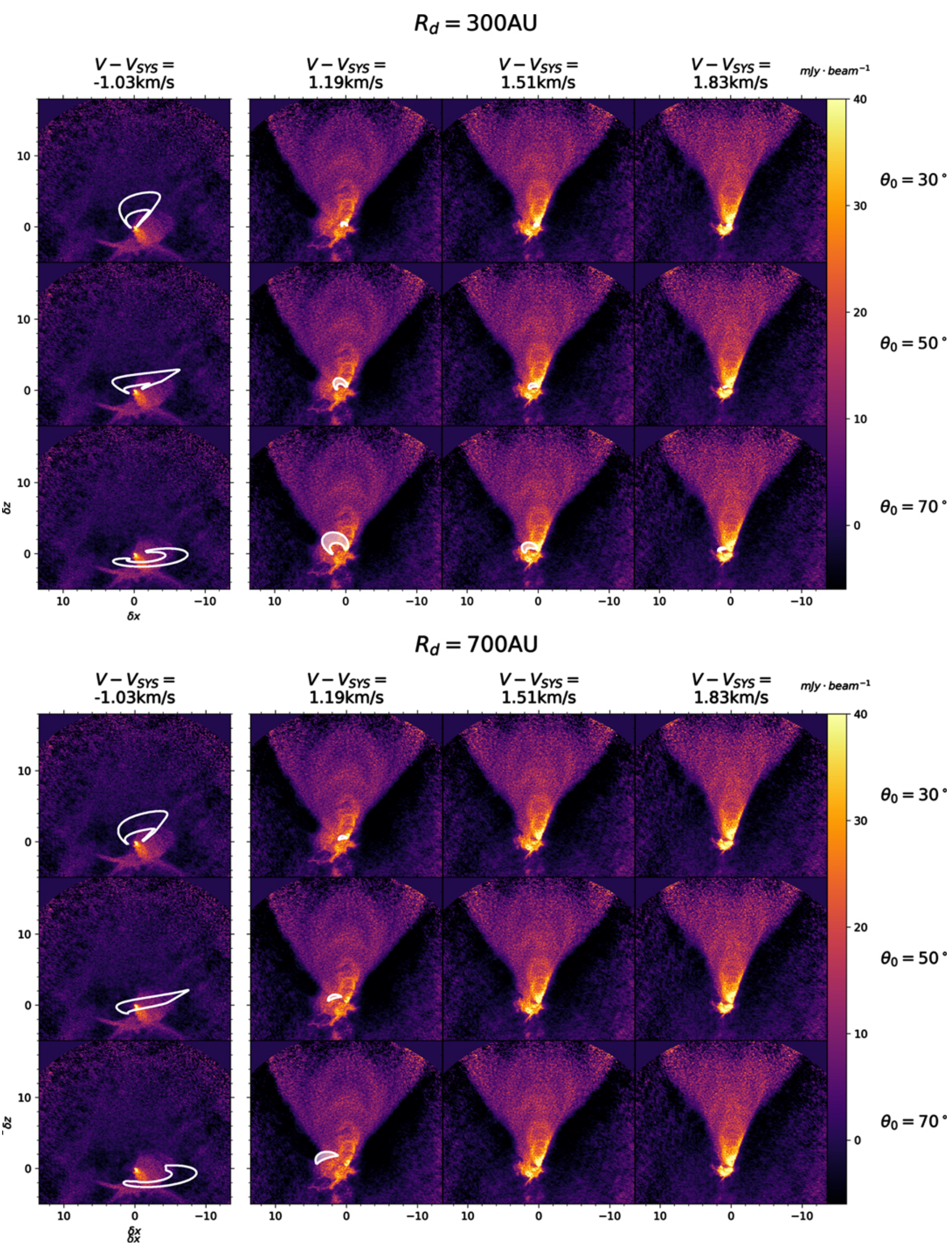

Fig. D.1. ${ }^{12} \mathrm{CO}$ selected individual channel maps. For each channel map $\Delta V=0.32 \mathrm{~km} \mathrm{~s}^{-1}$ and the central velocity is indicated. Similarly to Fig. 4 , the white contours trace the model of an infalling shell with $r_{\mathrm{d}}=300 \mathrm{au}($ top $), 700 \mathrm{au}$ (bottom), and $\theta_{0}=30^{\circ}, 50^{\circ}$, and $70^{\circ}$. 